\title{
Pieles Arquitectónicas Dinámicas. Prototipos a escala mediante prototipado rápido, microcontroladores y patrones plegados.
}

Dynamic architectural skins. Scale prototypes using rapid prototyping. microcontrollers and folding patterns.

\author{
> Mauro Chiarella \\ FADU-UNL/CONICET, Argentina \\ chiarell@fadu.unl.edu.ar
}

\author{
- Analia Raffin \\ FADU-UNL, Argentina \\ anaraffin@hotmail.com
}

\author{
> Silvina Lopaczek \\ FADU-UNL, Argentina \\ silvinalopaczek@hotmail.com \\ > Sebastian Martini \\ FADU-UNL, Argentina \\ sebamartinidis@gmail.com
}

\author{
> Nicolas Góngora \\ FADU-UNL, Argentina \\ nicfer22@gmail.com \\ > Francisco Bressan \\ FADU-UNL, Argentina \\ fbress@unl.edu.ar
}

\begin{abstract}
Contemporary architecture replaces the concept facade by skin: outer layer mediating between the building and its surroundings; active, informed, connected and communicative membrane. Our training and practice in architecture, still working for the rigor and stability of modular geomerías on determinants of a unique spatial and morphological situation passive. In the teaching of architecture in Latin America there are no exercises that incorporate a learning design to create flexible dynamic geometries as possible structures for sensitive skin. The research aims to explore the possibilities and systematize the dynamic folding of planar surfaces using geometric simulation programs (freeform origami, Grasshopper); building scale prototypes (rapid prototyping and arduino microcontroller) and subsequent verification of energy analysis program.
\end{abstract}

Keywords: architectural skin; folded patterns; arduino; rapid prototyping

\section{Introducción: del Muro a la Piel}

\section{"La arquitectura contemporánea sustituye la idea de fachada por la de piel: capa exterior mediadora entre el edificio y su entorno. No un alzado neutro sino una membrana activa, informada, comunicada y comunicativa. Más que meros agujeros, pieles técnicas interactivas. Pieles colonizadas por elementos funcionales capaces de alojar instalaciones y ser- vicios, capaces de captar y transmitir energías. Pero también capaces de soportar otras capas incorporadas: solapadas más que adheridas" Manuel Gauza}

Históricamente la arquitectura se ha caracterizado por generar espacialidades estables, sólidas, corpóreas. En las últimas décadas hay un marcado interés por abordar la pérdida de la materialidad y la incorporación de situaciones espaciales dinámicas, variables y flexibles. Al incorporar tecnologías innovadoras en la ideación, desarrollo y manufactura, es posible reinventar un clásico elemento constituyente de lo arquitectónico como es el muro. Es así como tradicionales características y objetivos referidos al muro están siendo redefinidos: lo estático se vuelve fluido, lo opaco se vuelve transparente, la barrera se vuelve filtro y los límites se vuelven neutrales. La tecnología ha permitido al muro convertirse en un dinámico y sensible componente del ambiente construido. Osman Ataman describe los avances alcanzados hasta hoy en la evolución de los sistemas de muros arquitectónicos que incorporan sensores en los edificios, combinando materiales, sistemas inteligentes, ingeniería $\mathrm{y}$ arte se pueden crear componentes que no solo soportan y definen un espacio sino que lo trasciende enriqueciéndolo. En la arquitectura contemporánea podemos clasificar, según sus cualidades y prestaciones: muros de geometrías curvas y complejas, muros dinámicos, muros responsivos, muros interactivos, muros inteligentes.

Entre los diferentes términos utilizados para describir la zona entre el exterior arquitectónico y su interior, existen ciertas diferencias entre lo que se conoce como: envolvente, pared, muro, piel y fachada. Envolvente es un término genérico que describe el cierre total de un edificio. Pared se ha utilizado tradicionalmente para describir una construcción de soporte vertical. Muro cortina es un término que aparece a principios del siglo XX para distinguir una construcción que no soporta carga. Piel es un término acuñado inicialmente para continuar con la distinción entre el revestimiento y la estructura de una pared, pero ha sido más recientemente asociada como un sistema ambiental inteligente. La fachada se asocia como parte de una composición urbana.

La evolución del Muro a la Piel Dinámica es aún una materia pendiente en la enseńanza de la arquitectura en Latinoamérica. Un muro podría ser más que una barrera o estructura, puede ser un sistema integrado y completo. Dentro de las prácticas y enseñanza arquitectónica observamos la ausencia de ejercicios proyectuales que incorporen estrategias didácticas para imaginar geometrías dinámicas, flexibles, como estructura posibilitante de las pieles sensibles propuestas. Nuestra formación y práctica disciplinar aún concibe geométricamente desde la rigurosidad y estabilidad modular que nos proponen las geomerías en un estado pasivo, determinante de una única situación espacial y 
morfológica. Nos preguntamos: ¿Cómo se pensaría la geometría de un muro de modo dinámico si convencionalmente se lo ha considerado como algo estable? A partir de esta problemática, nos interesa explorar la posibilidad de la incorporación estratégica de nuevas herramientas de diseño para la ideación, desarrollo y materialización de una piel arquitectónica dinámica, iniciando la exploración desde el plegado geométrico de superficies planares, pasando por la construcción de prototipos a escala mediante prototipado rápido y microcontroladores electrónicos y culminando en la verificación de valores con software de análisis energético.

\section{¿Pieles Reactivas o Responsivas?}

El diseño reactivo está vinculado a la activación por estímulos. Los sistemas reactivos son elementos de nuestro entorno que tienen (en diversos niveles de complejidad) una gama de contenidos predefinidos, como comportamientos que se pueden activar para producir un cambio. La mayor parte de diseńo de interacción hoy es una mejora estética de los modelos de diseño reactivo enraizada en los primeros métodos de instrucción en computación; un modelo rígido, lineal y algo restrictivo.

Ruairi Glynn sugiere que es más útil y productivo entender la interactividad como un modelo de dialogo o conversación, tal como naturalmente, ocurre entre los seres humanos o en el reino animal en general. La interactividad sería entonces como una actividad de conversación entre los usuarios y el objeto que se ha diseñado, en cambio un modelo reactivo funciona haciendo caso de rígidas restricciones previamente diseńadas e incorporadas en el objeto donde el comportamiento es orientado por los comandos conteniendo ciertas restricciones controladas preestablecidas. Michael Fox también argumenta a favor de la arquitectura dinámica en las cuales los sistemas tienen capacidad de aprendizaje y funcionan de acuerdo a un modelo conversacional. Fox (Kinetic Design Group, MIT) ha publicado los principios de los sistemas de comportamiento dinámicos identificando seis tipos ordenados por nivel de complejidad.

El concepto de responsivo se utiliza en un sentido general para describir una interacción entre un sistema dinámico y el entorno, incluye la interacción humana para generar una respuesta directa, además de reacción natural a una fuerza del medio ambiente aleatoria. De esta manera vemos que la interacción no se limita a los comportamientos rígidos de los sistemas reactivos. Como una extensión de esto, si a los artefactos diseñados se les da la capacidad de improvisar, negociar y aprender de ellos mismos, tienen el potencial, a través de la interacción con otros participantes de desarrollar sus propios comportamientos personales más allá de las nociones preconcebidas de su diseñador personal y así se convierten en objetos responsivos. Tal proceso evolutivo logra dotar a las pieles arquitectónicas de comportamientos y expresiones aumentando sus capacidades expresivas y de adaptación y eficiencia en relación a factores ambientales externos.

\section{Patrones Plegados}

Desde la antigüedad, la geometría se ha consolidado como el más poderoso instrumento para concebir y proyectar la arquitectura. La aplicación del universo geométrico en nuestra disciplina ha sido interpretada históricamente, como un recurso para garantizar la optimización de la forma; mejorar los patrones organizativos garantizando sistematicidad tecnológica y rigor constructivo. Aspectos funcionales, tecnológicos, simbólicos y culturales se sostienen desde lógicas geométricas que se irán modificando en coherencia con cambios y rupturas en nuestras formas de pensar, hacer y proyectar el ambiente según parámetros culturales y temporales.

Las composiciones plegadas en arquitectura emergieron como una nueva posibilidad proyectual, experimentada en diversas estrategias didácticas y obras contemporáneas: Vyzoviti 20032006-2011; Tachi 2009-2010; Saito 2005-2007-2012; Chiarella, 2006-2009-2011, en experiencias didácticas; Sancho-Madridejos, FOA, NOX, Toyo Ito, Herzog \& De Meuron, Rem Koolhas, en obras y proyectos entre otros. Una piel laminar plegable propone el desarrollo de patrones geométricos flexibles y dinámicos que van desde los cerramientos apoyados en nervaduras y estructuras reticuladas a diferentes membranas autoportantes. La base estructural de estas formas ha definido en la práctica estos dos modelos estratégicos de construcción: la primera está basada en el apoyo de los cerramientos sobre nervaduras como si fueran huesos estructurales y piel independiente (dECOi Architects, Aegis Hyposurface, 2001; Al Bahar Towers, 2012) y en la segunda se aprovecha la rigidez de las superficies envolventes como una construcción en dónde las piezas mismas pueden ser estructurales (Responsive Skin-maglab, 2012; Real Rigid Origami-T. Tachi, 2009). Mientras que en este último caso se explora las posibilidades de plegado de un mismo material continuo, el primero ejemplifica la combinación de procesos y materiales diversos. Los patrones plegados son a la vez una construcción formal, una acción operativa y una percepción sensible.

\section{Sistematización y clasificación de variables}

En la presente investigación, se han analizado y clasificado más de cuarenta composiciones plegadas según: parámetros característicos de los patrones, geometría base, materialidad posible, manipulabilidad-movimiento y grado de utilización para piel arquitectónica (morfología-espacialidad-tectónica). Las transformaciones geométricas de la superficie se originan con simples acciones, presentadas como una lista de verbos; plegar, presionar, arrugar, plisar o fruncir, marcar, cortar, arrancar, rotar, retorcer, girar, envolver, perforar, articular, anudar, entrelazar, comprimir, desplegar. En este sentido es sustancial caracterizar la geometría y vincularla con las condiciones físicas que permitan desarrollar la formulación, con posibilidades de ejecución en dimensiones arquitectónicas. Se considera que las propiedades de ejecución material son las determinantes en la definición de la forma final. Las propiedades materiales de las composiciones plegadas se pueden diferenciar primeramente entre los vértices (los dobleces o curvaturas de los planos) y las caras, y luego según su desarrollo. La caracterización material manifiesta las posibilidades de alternancia según los elementos utilizados y su conformación formal en distintas magnitudes, por lo que se considera que es fundamental realizar una experimentación progresiva de aplicaciones específicas, que vinculen la ideación y ejecución constructiva. Hacia esos objetivos se ha direccionado la construcción de seis prototipos dinámicos con sensores de movimiento e iluminación ambiental. 


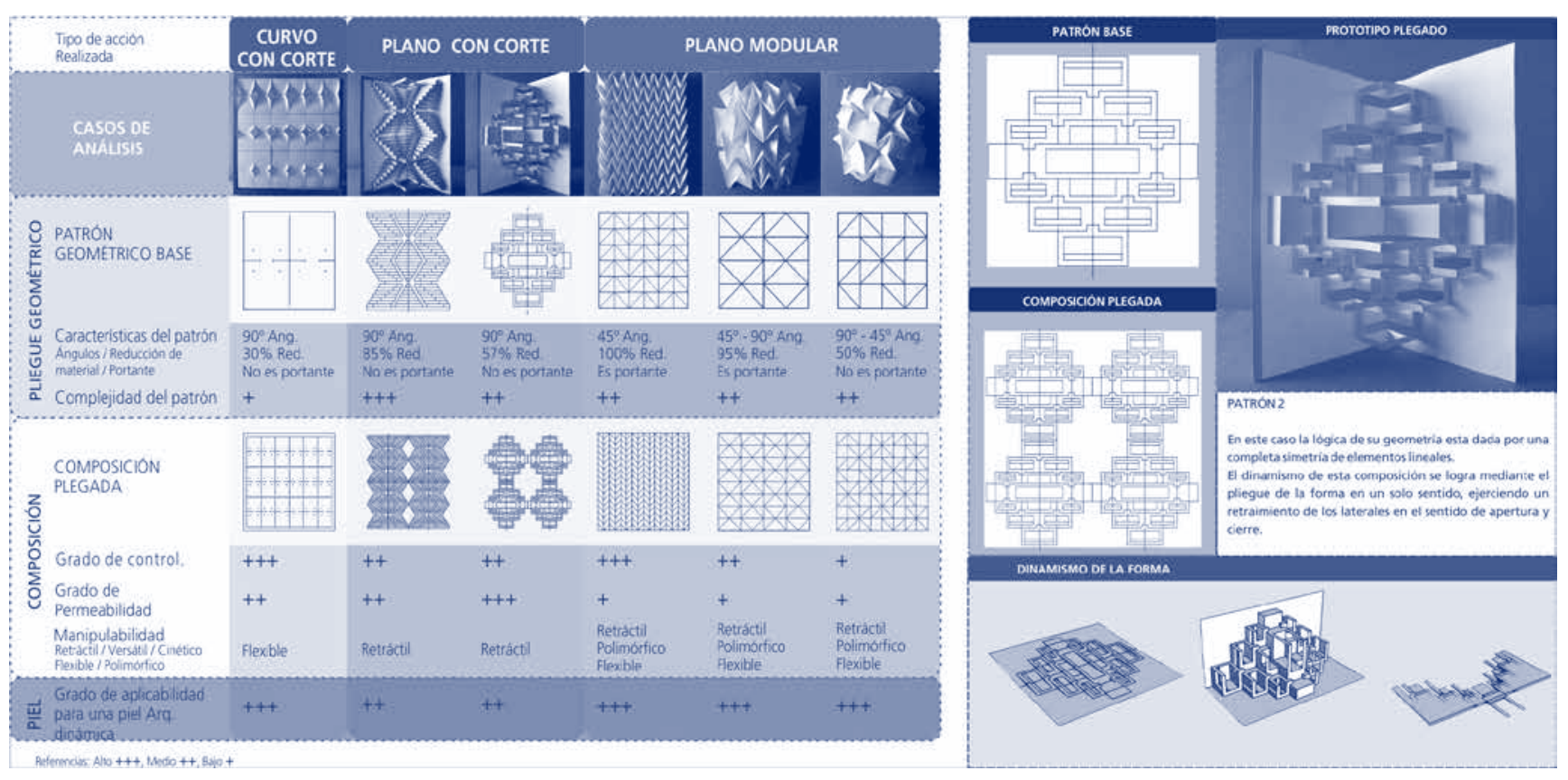

Figura 1: Análisis y clasificación de variables de composiciones plegadas (Cientibecaria UNL, A. Raffin).

\section{Data y Forma}

La potencialidad de estos desarrollos reside en definir la materialidad de la piel a partir de la definición de una geometría variable que responda a datos externos-internos y constituyan componentes activos por mecanismos electrónicos adjuntos. La incorporación de un microcontrolador electrónico (arduino) facilita el uso de la programación para proyectos multidisciplinares permitiendo la sincronización entre sensores, mecanismos físicos y parametrización geométrica conduciendo a un aumento en la eficiencia del diseño a través de la definición planificada del patrón geométrico a utilizar. Para el cambio de escala, la correcta elección de procesos simples de fabricación digital y diseño paramétrico estimula una innovación posible con un mayor control geométrico para la automatización de la manufactura de estos nuevos desafíos arquitectónicos.

\section{Simulación: microcontrolador + mecanismos}

Los sistemas complejos de plegados a su vez actúan utilizando placas articuladas, que permiten comportamientos cinéticos y dinámicos a través de mecanismos definidos por el tipo de matriz de pliegues. No sólo el plegado del material afecta a la integridad estructural del sistema, sino que también lo hace el espesor del material elegido en sí. Las características del material (flexibilidad, grosor, elasticidad, etc.) pueden restringir la traducción literal de un sistema de plegado a un soporte, ya que el comportamiento del material no se exterioriza de igual manera a cada tipo de manipulación. El potencial de no tener una estructura rígida complementaria amplia las múltiples posibilidades de superficie que el patrón otorga y es mucho más interesante para obtener diferentes formas deseadas. Sin embargo para poder lograr este dinamismo o mutabilidad es necesaria la participación de un controlador electrónico (arduino) el cual permite la oportunidad de un cambio de configuración superficial, que responde a los flujos de interacción externa al modelo. Un elemento que puede trabajar mecánica o automáticamente, haciendo que el sistema completo se comporte como un organismo vivo, capaz de asimilar y procesar información, en respuesta a un comportamiento emergente.

Luego de las primeras pruebas experimentales se empezó a idear los mecanismos que darían movimiento e interactividad a las composiciones, estudiando diversas posibilidades que estuvieran a nuestro alcance, así como también la forma de controlarlos. Esta exploración dio como resultado la implementación de servomecanismos como actuadores y sensores de proximidad ultrasónicos como elementos censantes del entorno, manejados a través de una programación diseñado a tal efecto. El kit electrónico utilizado se compone de 1 placa “Arduino Uno" Revisión 3, en el que se utilizaron 3 servomotores, 1 sensor de proximidad por ultrasonido HC-SR04, un diodo emisor de luz led y una resistencia de carbón limitadora de corriente. Cabe destacar que el soft utilizado para el diseño esquemático de conexiones es Fritzing (open-source).

Las etapas de desarrollo recorren diferentes instancias, en primer lugar aparece la Idea. Se realizaron experiencias de procesamiento de información tratando de entender la manera en que estaban generados estos modelos, cuál era el sentido en el que plegaban y la manera en que debían ser cortados. En segunda instancia se encentra el Diseño, en esta instancia se buscaba lograr una correlación entre la idea inicial y el paso a un modelo digital, de esta manera pusimos a prueba el rendimiento del diseńo. Y en tercer instancia se imprimió lo realizado digitalmente y se logró el modelo inicial que permitía realizar manipulaciones físicas para poder entender 


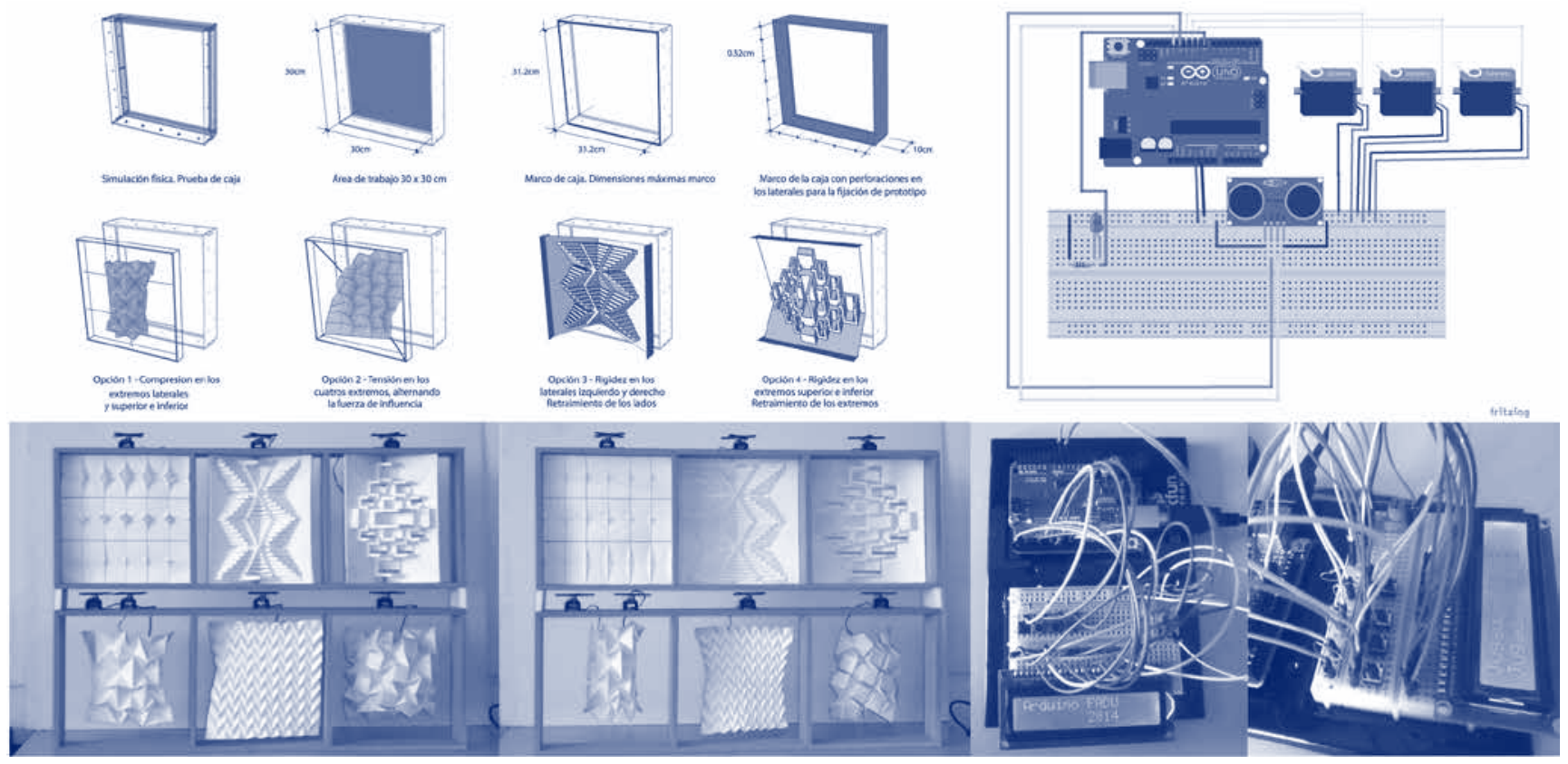

Figura 2: Prototipos a escala: microcontrolador + mecanismos (Grupo Arduino FADU: Est. Arq. N. Góngora: DI S. Martini; AIA F. Bressan)

su funcionamiento. En la cuarta fase digital, se llevó el modelo analógico a una interconexión directa con el modelado digital, para poder ajustar el funcionamiento interno de las partes, lo cual nos permitiría observar todo el dinamismo de la forma que en ciertos casos en lo analógico nos limitaba el tipo de material con el que habíamos realizado el modelo. En la quinta instancia se vincula el modelo físico con el sistema Arduino, este proceso nos brinda la oportunidad de vinculación analógico-digital, logrando así cumplir con el objetivo propuesto inicialmente.

Para el desarrollo de los prototipos, se realizó un diseño de caja contenedora, con un área de trabajo de $30 \times 30 \mathrm{~cm}$. Se pensaron cuatro modelos base de tensión (mecanismos) para los patrones. La primera es de compresión, tensando los laterales desde varios puntos para lograr que toda la forma se contraiga al mismo tiempo. La segunda opción tensa dos extremos contrarios de manera constante y contrae-retrae los extremos restantes. La opción tres y cuatro trabajan de manera similar, pero una con rigidez en los laterales y la otra en los extremos manteniéndolos fijos mediante se retrae el patrón. En nuestros prototipos, la regularidad geométrica permite diseñar el crecimiento del patrón en la dirección de sus ejes principales. Esta transformación de la superficie permite albergar pieles más flexibles o mutables. Al observar el comportamiento de estos modelos, se puede ver si los modelos logran el dinamismo necesario tomando en cuenta la posición de reposo y de tensión del material simulado.

Verificación: análisis energético (patrón y piel)

Utilizando el software Rhinoceros se generó el patrón mediante un conjunto de superficies que permitían manipular la composición plegada, adaptándola al modelo elegido. Luego se incorporó
Grasshopper para lograr una definición paramétrica que permita la reconstrucción del patrón geométrico para su posterior vinculación con el Software Ecotect. Grasshopper por su parte es un editor que permite el desarrollo de sistemas, usando un intuitivo método gráfico basado en un interface de nodos, el usuario define una secuencia de instrucción que se convierte en modelos tridimensionales en la ventana de Rhino 3D. Mediante la utilización del programa Ecotect conseguimos realizar una evaluación solar de la superficie, encontrando la posición óptima para su posterior ejecución y entendiendo cómo funciona la piel propuesta. A través el plug-in Geco de Grasshopper, conseguimos la conexión del modelo parametrizado de la envolvente con los análisis de Ecotect, de modo que el programa podrá realizar los cálculos para determinar el posicionamiento del sol en relación a al modelo elegido, para obtener condiciones óptimas para los ambientes a diseñar. El objetivo de esta etapa es verificar y definir los términos de referencia desde el punto de vista ambiental que podrán ser incorporados al modelo de composición plegado seleccionado para su evaluación.

\section{Conclusiones}

Una piel dinámica puede concebirse a través del diseño de geometrías logradas mediante la manipulación y su programación permitiendo descubrir la potencialidad propia de sus superficies, llevando a cabo la materialización de los modelos a escala aplicando prototipado rápido (cortadora laser) para operar con materiales laminares simples. La incorporación de composiciones plegadas a través de patrones geométricos con potencial de tornarse responsivos, permite ampliar los recursos proyectuales para la ideación, desarrollo y materialización de una piel arquitectónica dinámica. La implementación de prototipos a escala en las disciplinas proyectuales 

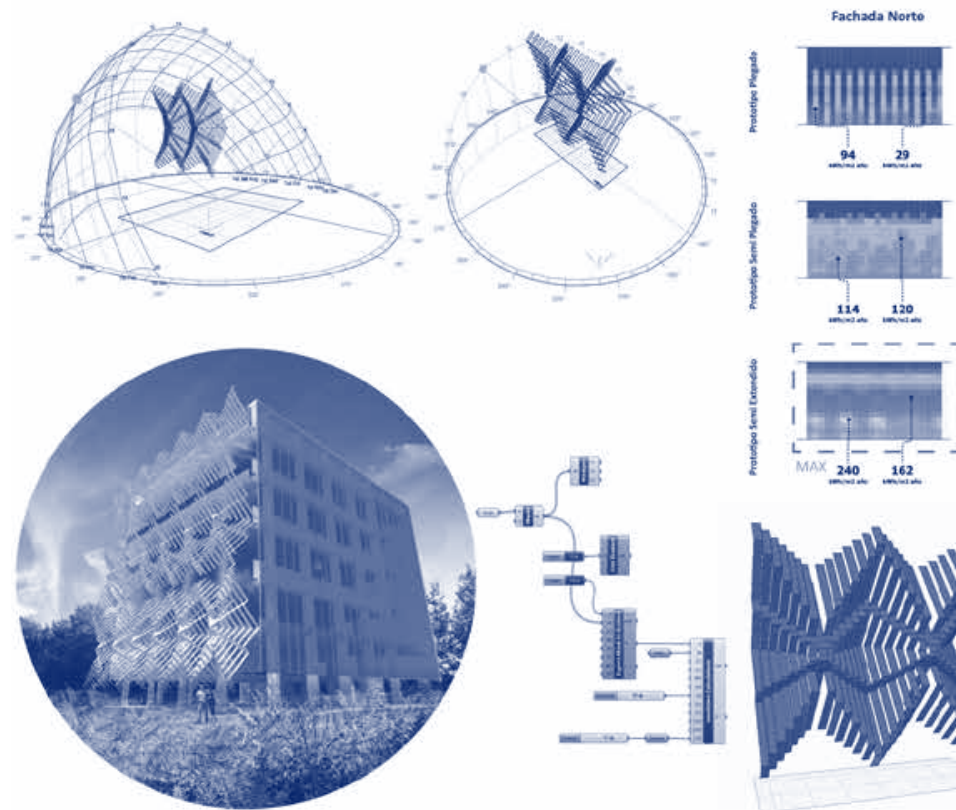

$\ldots$
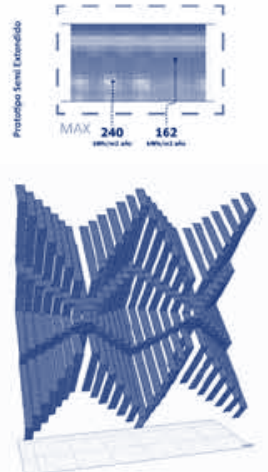
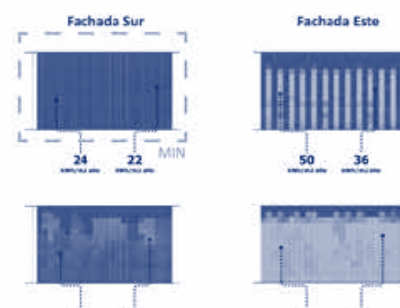

so. $\quad 36$

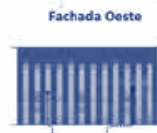

$+48$
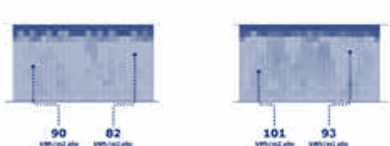

101
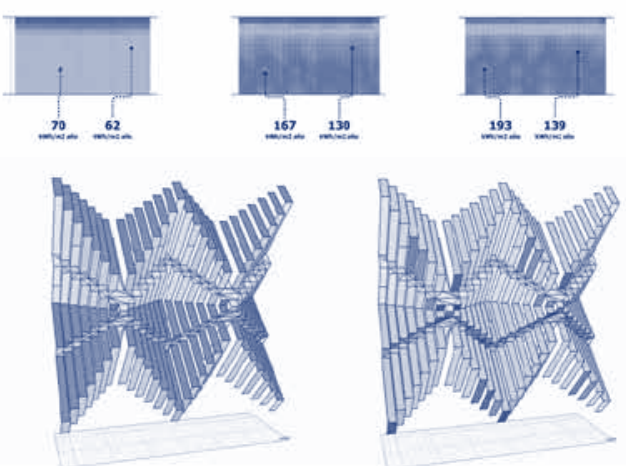

Figura 3 : Evaluación solar de superficies. Patrón plegado y piel en 3 situaciones de movimiento (propuesta edificio anexo FADU-UNL).

es un recurso que permite evaluar las posibilidades del material y los posibles problemas de construcción y de mecanismos utilizados, de allí radica la importancia en la selección de los mismos, técnicas y estudio de detalles constructivos, concibiéndose este proceso no solo como una herramienta de acompañamiento del proyecto, sino como una práctica formal en sí misma.

La colaboración de disciplinas ajenas a la arquitectura (analista de informática aplicada, diseñador industrial) ha permitido abordar los comportamientos de la programación para el caso de cada prototipo con capacidad de respuesta en tiempo real a parámetros de iluminación y movimiento. Este tipo de desarrollos experimentales participa en esta nueva etapa de hibridación inherente a las tendencias de la creación contemporánea, en la que los proyectos de desarrollo se han desplazado de lo individual a una práctica de conjunto. Es decir, una comunidad multidisciplinar de creadores, donde el trabajo colectivo es una prioridad, centrándose en la naturaleza colaborativa propia de los procesos proyectuales actuales.

\section{Agradecimientos}

A los profesores, docentes y ayudantes CID-FADU-UNL: M. Dalla Costa; Alfredo Stipech; M. Elena Tosello; Georgina Bredanini y Cecilia Zorzón. PACT No1/CAID2011-Chiarella.

\section{Referencias}

Chiarella, M; Garcia Alvarado, R. Composiciones Plegadas. Propiedades espaciales y materiales (envolventes y componentes). SIGraDi 2012, Fortaleza. Brasil

Gausa, M. Diccionario metápolis de Arquitectura Avanzada. Barcelona, Actar, 2001.

Glynn, R. Conversational environments Revisited, Bartlett School of Architecture, UCL, Wates house, Londres. Cybernetic conference, 2008.

Osman, A; Rogers, J; Ilesanmi, A. Redefining the Wall: Architecture, Materials and Macroelectronics. International Journal of Architectural Computing 4, no. 4 (2006): 125-136.

Vyzoviti, S. Out of the box and into the fold. Der Große Wurf. Faltungen in Der Gegenwartskunst Kunstmuseen Krefeld = Falling right into place: the fold in contemporary art, Freiburg, 2008 (34-42) 